\title{
Quasi-Fibonacci oscillators
}

\author{
A.M. Gavrilik, I.I. Kachurik, A.P. Rebesh \\ Bogolyubov Institute for Theoretical Physics, Kiev 03680, Ukraine \\ E-mail: omgavr@bitp.kiev.ua
}

\begin{abstract}
We study the properties of sequences of the energy eigenvalues for some generalizations of $q$-deformed oscillators including the $p, q$-oscillator, the 3 -, 4 - and 5-parameter deformed oscillators given in the literature. It is shown that most of the considered models belong to the class of so-called Fibonacci oscillators for which any three consecutive energy levels satisfy the relation $E_{n+1}=\lambda E_{n}+\rho E_{n-1}$ with real constants $\lambda, \rho$. On the other hand, for certain $\mu$-oscillator known from 1993 we prove the fact of its non-Fibonacci nature. Possible generalizations of the three-term Fibonacci relation are discussed among which we choose, as most adequate for the $\mu$-oscillator, the so-called quasi-Fibonacci (or local Fibonacci) property of the energy levels. The property is encoded in the three-term quasiFibonacci $(\mathrm{QF})$ relation with non-constant, $n$-dependent coefficients $\lambda$ and $\rho$. Various aspects of the QF relation are elaborated for the $\mu$-oscillator and some of its extensions.
\end{abstract}

PACS numbers: 02.20.Uw, 03.65.-w, 03.65.Ge, 03.65.Fd, 05.30.Pr

\section{Introduction}

In 1991, the two-parameter family of $p, q$-deformed quantum oscillators has been introduced [1]. Soon after, in [2] the family was named "Fibonacci oscillators", due to the basic property of the family encoded in the three-term linear recurrence relation [2]

$$
E_{n+1}=\lambda E_{n}+\rho E_{n-1}, \quad n \geq 1, \quad \lambda, \rho \in \mathbb{R}
$$

for any three consecutive values of energy levels from the spectrum of respective deformed quantum oscillators, with $\lambda$ and $\rho$ definite coefficients that depend on specific model of deformed oscillator. The usual Fibonacci numbers form the sequence generated by equation like (1) in which $\lambda=\rho=1$ and the first two members of the sequence are fixed as 1 and 1; the property states that each number in the sequence results from the sum of two preceding ones.

Following [2], in our paper we call the Fibonacci oscillators those oscillators whose any three energies $E_{n-1}, E_{n}, E_{n+1}$ satisfy the Fibonacci relation (FR) given by (1). They form the Fibonacci class of oscillators. There exist a number of known oscillators that are the Fibonacci ones. For instance, usual harmonic oscillator certainly is the Fibonacci oscillator in the sense of eq. (1) with $\lambda=2$ and $\rho=-1$. As already mentioned, the family of $p, q$-oscillators which contains well-known models of one-parameter $q$-oscillators, belongs to the Fibonacci class. However, the question naturally arises about possible existence of other models of deformed oscillators which satisfy (1), i.e., 
possess the Fibonacci property (FP). Recently, in conjunction with generalized Heisenberg algebras [3] possible extensions of the FP were studied, either in the direction of nonlinearization of the relation, or towards so-called $k$-step extension (in particular, Tribonacci sequence), see [4]. Also, in ref. [5] some classes of non-Fibonacci (namely so-called $k$-bonacci) oscillators have been explored.

Our goal in this paper is two-fold. First, we study from the viewpoint of possessing the FP some multi-parameter extended families of deformed oscillators, including 3and 4-parametric ones from $[6,7,8]$ as well as their 5-parameter extended model given in [9]. We prove that all these multi-parameter deformations belong to the class of Fibonacci oscillators. On the other hand, we examine certain as yet not well studied deformation called $\mu$-oscillator, which appeared in [10], and present the proof that this model lives outside the Fibonacci class (that is, it is non-Fibonacci one). This conclusion served for us as motivation to explore possible (still linear) extensions ${ }^{1}$ of the FR and to find among them most adequate and natural form of generalization which is suitable for the $\mu$-oscillator. In other words, our second goal is to provide a proper generalization of the FP that is adequate for both the $\mu$-oscillator and its several multiparameter extensions. The employed particular generalization of the FP can be termed "quasi-Fibonacci" property. Correspondingly, the $\mu$-oscillator [10] and its appropriate extensions belong to the class of quasi-Fibonacci (QF) oscillators. In some sense, this can also be viewed as the deformation of quantum oscillator whose energy values form a "locally-Fibonacci" sequence of values.

\section{Deformed oscillators of the Fibonacci class}

In this section we examine the energy spectra of the models of multi-parameter deformed oscillators from $[6,7,8,9]$, from the viewpoint of possessing the FP. But first it will be useful to remind main facts about some well-known models of deformed oscillators. General approach for treatment of deformed oscillators involves the notion $[12,13,14]$ of the structure function $f(N)$ or $\varphi(N)$ that serves to define the deformation. In terms of the structure function $\varphi(N)$ given by the equalities

$$
\varphi(N)=a^{\dagger} a, \quad \varphi(N+1)=a a^{\dagger}
$$

the most general commutation relation can be written in the form

$$
\varphi(N+1)-F(N) \varphi(N)=G(N)
$$

with $F(N)$ and $G(N)$ real functions. Every special choice of $F(N)$ and $G(N)$ provides, through solving the latter equation [13], the particular explicit form of the structure function $\varphi(N)$ and thus the corresponding model of deformed oscillator. With fixed $\varphi(N)$, the commutation relations for the generating elements $a, a^{\dagger}$ and $N$ of the concrete model of deformed oscillator are given as

$$
\begin{aligned}
& {\left[N, a^{\dagger}\right]=a^{\dagger}, \quad[N, a]=-a,} \\
& a a^{\dagger}-a^{\dagger} a=\varphi(N+1)-\varphi(N) .
\end{aligned}
$$

\footnotetext{
${ }^{1}$ For comparison see e.g. ref. [11] for an interesting example of nonlinear three-term relation giving the values of (non-equidistant) area spectrum of 5-dim Gauss-Bonnet black hole.
} 
That means we use the form of basic commutation relation such that $F(N)=1$ and $G(N)=\varphi(N+1)-\varphi(N)$. In this case, fixation of the structure function $\varphi(N)$ completely specifies the (deformed oscillator) model.

\section{$2.1 \quad$ Well-known Fibonacci oscillators}

Let us recall some most popular models of deformed oscillators which involve one or two deformation parameters and belong to the Fibonacci class of oscillators.

- Arik-Coon (AC) model [15]

This most early known model is given by the commutation relations (3) and

$$
a a^{\dagger}-q a^{\dagger} a=1
$$

In what follows, we use for all the models the same definition of the Hamiltonian as for the usual harmonic oscillator:

$$
H=\frac{1}{2}\left(a a^{\dagger}+a^{\dagger} a\right)
$$

where $\hbar \omega=1$ is meant. Using the appropriately modified (deformed) version of the Fock space $[13,14]$ wherein $H|n\rangle=E_{n}|n\rangle$, and $\varphi_{A C}(N)|n\rangle=\varphi_{A C}(n)|n\rangle$, the energy spectrum of AC model is

$$
E_{n}=\frac{1}{2}\left(\varphi_{A C}(n+1)+\varphi_{A C}(n)\right)=\frac{1}{2}\left([n+1]_{A C}+[n]_{A C}\right)=\frac{1}{2}\left(\frac{q^{n+1}-1}{q-1}+\frac{q^{n}-1}{q-1}\right)
$$

where the definition of the $A C$-type $q$-bracket (for $X$ either a number or an operator) and the structure function is given as

$$
[X]_{A C}=\frac{q^{X}-1}{q-1} \quad \Leftrightarrow \quad \varphi_{A C}(n)=\frac{q^{n}-1}{q-1}=[n]_{A C}
$$

so that the latter satisfies (5).

One can easily check that the AC-oscillator energies (7) satisfy the FR (1) if $\lambda=1+q$ and $\rho=-q$.

- Biedenharn-Macfarlane (BM) model [16, 17]

The model is determined by the commutation relations (3) and the relations

$$
a a^{\dagger}-q a^{\dagger} a=q^{-N}, \quad a a^{\dagger}-q^{-1} a^{\dagger} a=q^{N} .
$$

The corresponding structure function which solves (9) is

$$
\varphi_{B M}(N)=\frac{q^{N}-q^{-N}}{q-q^{-1}} \equiv[N]_{B M}
$$

and the energy spectrum reads

$$
E_{n}=\frac{1}{2}\left(\varphi_{B M}(n+1)+\varphi_{B M}(n)\right)=\frac{1}{2}\left(\frac{q^{n+1}-q^{-(n+1)}}{q-q^{-1}}+\frac{q^{n}-q^{-n}}{q-q^{-1}}\right) .
$$


The BM oscillator satisfies the FR (1) with $\lambda=q+q^{-1}$ and $\rho=-1$.

- $p, q$-deformed oscillator [1]

This model is determined by the commutation relations (3) and the relations

$$
a a^{\dagger}-q a^{\dagger} a=p^{N}, \quad a a^{\dagger}-p a^{\dagger} a=q^{N},
$$

possessing the $q \leftrightarrow p$ symmetry. The relevant structure function satisfying (12) is

$$
\varphi_{p, q}(N)=\frac{q^{N}-p^{N}}{q-p} \equiv[N]_{p, q}
$$

and hence the energy spectrum reads

$$
E_{n}=\frac{1}{2}\left(\frac{q^{n+1}-p^{n+1}}{q-p}+\frac{q^{n}-p^{n}}{q-p}\right) .
$$

The model of $p, q$-deformed oscillator is popular enough: it finds a number of interesting applications, see e.g. $[18,19,20,21,22,23,24,25]$. From it, by setting $p=1$ the AC-model is retrieved. At $p=q^{-1}$ we recover the BM-type oscillator. Finally, the peculiar case of $p=q$ corresponds to the so-called Tamm-Dancoff (TD) deformed oscillator $[26,27]$. This model, though not so popular as the former two, nevertheless possesses some interesting properties. More details concerning the TD-type oscillator model including some of its properties can be found in the paper [28]. Note that the TD oscillator arises also as a particular one-dimensional case in the $S U(d)$ covariant $d$-dimensional so-called $q$-deformed Newton oscillator [29].

With (14), the two-parameter $p, q$-model of deformed oscillators satisfies the FR (1) for $\lambda=q+p$ and $\rho=-q p$. Accordingly, the AC-oscillator is the Fibonacci oscillator with $\lambda=1+q$ and $\rho=-q$, whereas the energy levels of the BM-oscillator satisfy (1) if we take $\lambda=q+q^{-1}$ and $\rho=-1$. Finally, to confirm that the TD-model of deformed oscillator does satisfy (1), we put $\lambda=2 q$ and $\rho=-q^{2}$.

\section{$2.2 \quad$ A family of multi-parameter deformed oscillators}

In this subsection we focus on those deformed multi-parameter oscillators which possess more then two deformation parameters in their defining relations and thus generalize the $q$ - and $p, q$-deformed oscillators. It is of special interest to examine the properties of such deformed oscillators in dependence on the number of parameters. Our main goal is to examine whether these models of oscillators satisfy the FR (1) and in case they don't, to search for a way of appropriately modifying the FR.

As the example of multi-parameter oscillators consider the $(p, q, \alpha, \beta, l)$-deformed oscillator family formulated in [9] and given by the defining relations

$$
a a^{\dagger}-q^{l} a^{\dagger} a=p^{-\alpha N-\beta}, \quad a a^{\dagger}-p^{-l} a^{\dagger} a=q^{\alpha N+\beta} .
$$

Here $p, q, \alpha, \beta, l$ are the parameters of deformation. Creation and annihilation operators act upon the Fock space basis state $|n\rangle$ by the formulas

$$
a a^{\dagger}|n\rangle=\frac{q^{\alpha n+\beta+l}-p^{-(\alpha n+\beta+l)}}{q^{l}-p^{-l}}|n\rangle, \quad a^{\dagger} a|n\rangle=\frac{q^{\alpha n+\beta}-p^{-(\alpha n+\beta)}}{q^{l}-p^{-l}}|n\rangle .
$$


With the Hamiltonian as in (6) the eigenvalues which form the energy spectrum are

$$
E_{n}=\frac{1}{2}\left(\frac{q^{\alpha n+\beta+l}-p^{-(\alpha n+\beta+l)}}{q^{l}-p^{-l}}+\frac{q^{\alpha n+\beta}-p^{-(\alpha n+\beta)}}{q^{l}-p^{-l}}\right) .
$$

Unlike the models mentioned above, for this 5-parameter model the formula for $E_{n}$ cannot be written as $E_{n}=\frac{1}{2}\left(\varphi_{n+1}+\varphi_{n}\right)$, but rather as $E_{n}=\frac{1}{2}\left(\varphi_{n}+\varphi_{n+\frac{l}{\alpha}}\right)$; only in the 4-parameter case wherein $l=\alpha$ the presentation $E_{n}=\frac{1}{2}\left(\varphi_{n}+\varphi_{n+1}\right)$ is retrieved.

The models of deformed oscillators examined in Subsection 2.1 obviously follow from the $(p, q, \alpha, \beta, l)$-deformed oscillator as particular cases . Moreover, the 5-parameter family reduces at $l=1$ to the 4-parameter deformed oscillator treated in Ref. $[7,8]$ which in its turn goes over into the three-parameter deformed quantum oscillators first formulated in [6].

\subsection{Fibonacci property of multi-parameter oscillator models}

The constants $\lambda$ and $\rho$ in (1) for the AC, BM and $p, q$-models of deformed oscillators given in Subsection 2.1 can be deduced easily by solving the corresponding systems of equations. The principal feature of those models is that the coefficients $\lambda, \rho$ are indeed constant, i.e., they do not depend on the eigenvalue $n$ of the (particle number) operator $N$. Suppose that (1) is true for the extended 3-, 4- and 5-parameter oscillators. Then the following statement is true.

Proposition 1. The five-parameter deformed oscillator whose energies $E_{n}, n \geq 0$, are given by (16), satisfies, with certain constants $\lambda, \rho$ each of the following two relations

$$
\left\{\begin{array}{l}
E_{n+1}=\lambda E_{n}+\rho E_{n-1} \\
E_{n+2}=\lambda E_{n+1}+\rho E_{n}
\end{array}\right.
$$

and thus belongs to the class of Fibonacci oscillators.

The proof proceeds through solving for $\lambda$ and $\rho$ the system (17) with account of (16). Recall, these $\lambda$ and $\rho$ should not depend on $n$. As follows from (17),

$$
\lambda=\frac{E_{n+2} E_{n-1}-E_{n+1} E_{n}}{E_{n+1} E_{n-1}-E_{n}^{2}}, \quad \rho=\frac{E_{n+1}^{2}-E_{n+2} E_{n}}{E_{n+1} E_{n-1}-E_{n}^{2}} .
$$

Inserting from (16) the expression for $E_{j}, j=n-1, n, n+1, n+2$, we obtain $\lambda=B / C$ where $B=B(n ; p, q, \alpha, \beta, l), C=C(n ; p, q, \alpha, \beta, l)$, and

$$
\begin{aligned}
B & =\left(\frac{q^{\alpha(n+2)+\beta+l}-p^{-\alpha(n+2)-\beta-l}+q^{\alpha(n+2)+\beta}-p^{-\alpha(n+2)-\beta}}{q^{l}-p^{-l}}\right) \times \\
& \times\left(\frac{q^{\alpha(n-1)+\beta+l}-p^{-\alpha(n-1)-\beta-l}+q^{\alpha(n-1)+\beta}-p^{-\alpha(n-1)-\beta}}{q^{l}-p^{-l}}\right)- \\
& -\left(\frac{q^{\alpha(n+1)+\beta+l}-p^{-\alpha(n+1)-\beta-l}+q^{\alpha(n+1)+\beta}-p^{-\alpha(n+1)-\beta}}{q^{l}-p^{-l}}\right) \times \\
& \times\left(\frac{q^{\alpha n+\beta+l}-p^{-\alpha n-\beta-l}+q^{\alpha n+\beta}-p^{-\alpha n-\beta}}{q^{l}-p^{-l}}\right),
\end{aligned}
$$




$$
\begin{aligned}
C & =\left(\frac{q^{\alpha(n+1)+\beta+l}-p^{-\alpha(n+1)-\beta-l}+q^{\alpha(n+1)+\beta}-p^{-\alpha(n+1)-\beta}}{q^{l}-p^{-l}}\right) \times \\
& \times\left(\frac{q^{\alpha(n-1)+\beta+l}-p^{-\alpha(n-1)-\beta-l}+q^{\alpha(n-1)+\beta}-p^{-\alpha(n-1)-\beta}}{q^{l}-p^{-l}}\right)- \\
& -\left(\frac{q^{\alpha n+\beta+l}-p^{-\alpha n-\beta-l}+q^{\alpha n+\beta}-p^{-\alpha n-\beta}}{q^{l}-p^{-l}}\right)^{2} .
\end{aligned}
$$

Calculation shows remarkable cancelation of $n$-dependence: we get $B / C=B^{\prime} / C^{\prime}$ where

$$
\begin{aligned}
B^{\prime} & =-q^{3 \alpha} p^{2 \alpha+l}-q^{3 \alpha} p^{2 \alpha}-q^{3 \alpha+l} p^{2 \alpha+l}-q^{3 \alpha+l} p^{2 \alpha}-p^{-\alpha}-p^{-\alpha+l}-q^{l} p^{-\alpha}- \\
& -q^{l} p^{-\alpha+l}+q^{\alpha} p^{l}+q^{\alpha}+q^{\alpha+l} p^{l}+q^{\alpha+l}+q^{2 \alpha} p^{\alpha+l}+q^{2 \alpha} p^{\alpha}+q^{2 \alpha+l} p^{\alpha+l}+q^{2 \alpha+l} p^{\alpha} \\
C^{\prime} & =-p^{l}-1-q^{l} p^{l}-q^{l}+2 q^{\alpha} p^{\alpha+l}+2 q^{\alpha} p^{\alpha}+2 q^{\alpha+l} p^{\alpha+l}+ \\
& +2 q^{\alpha+l} p^{\alpha}-q^{2 \alpha} p^{2 \alpha+l}-q^{2 \alpha} p^{2 \alpha}-q^{2 \alpha+l} p^{2 \alpha+l}-q^{2 \alpha+l} p^{2 \alpha} .
\end{aligned}
$$

Moreover, it is easy to check that $\left(q^{\alpha}+p^{-\alpha}\right) C^{\prime}=B^{\prime}$. Hence finally

$$
\lambda=q^{\alpha}+p^{-\alpha}
$$

The same procedure applied to $\rho$ yields $\rho=D / E$ where

$$
\begin{aligned}
D= & q^{3 \alpha} p^{\alpha}+q^{3 \alpha+l} p^{\alpha}+q^{\alpha} p^{-\alpha}+q^{\alpha} p^{-\alpha+l}+q^{\alpha+l} p^{-\alpha}+q^{\alpha+l} p^{-\alpha+l}- \\
& -2 q^{2 \alpha} p^{l}-2 q^{2 \alpha}-2 q^{2 \alpha+l} p^{l}-2 q^{2 \alpha+l}+q^{3 \alpha} p^{\alpha+l}+q^{3 \alpha+l} p^{\alpha+l}, \\
E= & -p^{l}-1-q^{l} p^{l}-q^{l}+2 q^{\alpha} p^{\alpha+l}+2 q^{\alpha} p^{\alpha}+2 q^{\alpha+l} p^{\alpha+l}+ \\
& +2 q^{\alpha+l} p^{\alpha}-q^{2 \alpha} p^{2 \alpha+l}-q^{2 \alpha} p^{2 \alpha}-q^{2 \alpha+l} p^{2 \alpha+l}-q^{2 \alpha+l} p^{2 \alpha},
\end{aligned}
$$

and it is easily checked that $D=\left(-q^{\alpha} p^{-\alpha}\right) E$. As result,

$$
\rho=-q^{\alpha} p^{-\alpha}
$$

So we conclude: thus found $\lambda$ and $\rho$ in (19) and (20) do solve (17), and do not depend on $n$. Hence we have proven the Proposition.

\section{A case for generalizing the Fibonacci property}

In this section we will examine, from the viewpoint of (non)validity of FP, the $\mu$-deformed oscillator formulated in [10]. We will prove that the FP is not valid in that case. Then our next goal is to find generalization of the FP appropriate for the $\mu$-oscillator. For proper extension, we adopt the so-called "quasi-Fibonacci" (QF) property encapsulated in the QF relation satisfied by members of the QF sequence. But, first let us recall necessary setup of the $\mu$-deformed oscillator. 


\subsection{Nonlinear $\mu$-deformed oscillator}

The $\mu$-oscillator [10] is defined in terms of the unital algebra whose generating elements $a, a^{\dagger}$ and $N$ satisfy

$$
\begin{aligned}
& {[N, a]=-a, \quad\left[N, a^{\dagger}\right]=a^{\dagger}, \quad\left[N, a a^{\dagger}\right]=\left[N, a^{\dagger} a\right]=0,} \\
& a a^{\dagger}-a^{\dagger} a=\varphi_{\mu}(N+1)-\varphi_{\mu}(N),
\end{aligned}
$$

and its structure function is given as

$$
a^{\dagger} a=\varphi_{\mu}(N) \equiv \frac{N}{1+\mu N}, \quad a a^{\dagger}=\varphi_{\mu}(N+1) \equiv \frac{N+1}{1+\mu(N+1)}
$$

with $\mu$ a deformation parameter. That is, the basic commutation relations for the $\mu$-oscillator are eq. (3) and

$$
\left[a, a^{\dagger}\right]=\frac{N+1}{1+\mu(N+1)}-\frac{N}{1+\mu N} .
$$

In the $\mu$-deformed version of Fock space, with normalized ground state $|0\rangle$ such that

$$
a|0\rangle=0, \quad N|0\rangle=0, \quad \varphi_{\mu}(0)=0,
$$

we have the infinite set of basis states

$$
|n\rangle=\frac{\left(a^{\dagger}\right)^{n}}{\sqrt{\varphi_{\mu}(n) !}}|0\rangle, \quad\langle n \mid m\rangle=\delta_{m n}, \quad n, m=0,1,2, \ldots,
$$

$\varphi_{\mu}(n) !=\varphi_{\mu}(n) \varphi_{\mu}(n-1) \ldots \varphi_{\mu}(1)$ on which

$$
\begin{aligned}
& N|n\rangle=n|n\rangle, \quad \varphi_{\mu}(N)|n\rangle=\varphi_{\mu}(n)|n\rangle, \\
& \langle n-1|a| n\rangle=\left\langle n\left|a^{\dagger}\right| n-1\right\rangle=\sqrt{\varphi_{\mu}(n)}=\left(\frac{n}{1+\mu n}\right)^{\frac{1}{2}} .
\end{aligned}
$$

Since

$$
\varphi_{\mu}(n)=\frac{n}{1+\mu n}
$$

the energy spectrum of this model reads

$$
E_{n}=\frac{1}{2}\left(\varphi_{\mu}(n+1)+\varphi_{\mu}(n)\right)=\frac{1}{2}\left(\frac{n+1}{1+\mu(n+1)}+\frac{n}{1+\mu n}\right)
$$

where the deformation parameter $\mu$ is assumed to satisfy $\mu \geq 0$. Setting $\mu=0$ recovers the known formulae for the standard quantum harmonic oscillator. 


\subsection{Non-Fibonacci nature of the $\mu$-oscillator}

Suppose the $\mu$-oscillator, with $\varphi(N)$ given in (21), obeys the FP stating that

$$
E_{n+1}-\lambda E_{n}-\rho E_{n-1}=0
$$

with constant $\lambda$ and $\rho$. With account of (26) this rewrites as

$\frac{n+2}{1+\mu(n+2)}+\frac{n+1}{1+\mu(n+1)}-\lambda\left(\frac{n+1}{1+\mu(n+1)}+\frac{n}{1+\mu n}\right)-\rho\left(\frac{n}{1+\mu n}+\frac{n-1}{1+\mu(n-1)}\right)=0$.

Then the following statement is true.

Proposition 2. The $\mu$-oscillator is not the Fibonacci one, i.e., (28) fails for it.

To prove, we take into account the equality, stemming from (28), of the two polynomials one of which is zero. This leads to the following set of equations:

$$
\begin{array}{ll}
n^{4}: & -1+\lambda+\rho=0 ; \\
n^{3}: & \lambda(3+2 \mu)+\rho(3+2 \mu)-3-2 \mu=0 ; \\
n^{2}: & \lambda\left(6+9 \mu-2 \mu^{2}\right)+\rho\left(6+7 \mu-2 \mu^{2}\right)-6-11 \mu+2 \mu^{2}=0 ; \\
n^{1}: & \lambda\left(\mu^{2}\left(\mu^{-1}-1\right)+\left(\mu^{-1}+2\right)\left(2 \mu^{-2}+\mu^{-1}-2\right)\right)+\rho\left(-\mu^{2}\left(\mu^{-1}+1\right)+\right. \\
& \left.+\left(\mu^{-1}+2\right)\left(2 \mu^{-2}-\mu^{-1}-2\right)\right)-2-10 \mu+\mu^{2}+4 \mu^{3}=0 ; \\
n^{0}: & 2 \mu^{2}-2+\mu(1+2 \mu)\left[(\lambda-1)\left(\mu^{-1}-1\right)+\rho\left(\mu^{-1}+1\right)\right]=0 .
\end{array}
$$

We have to solve this set for $\lambda$ and $\rho$. The first two equations yield $\lambda+\rho=1$. However, insertion this in the rest of equations leads us to inconsistency, as there is no solution satisfying the whole system, for arbitrary $\mu$. So we conclude: the $\mu$-oscillator with $\varphi(n)$ and $E_{n}$ from (25)-(26) does not possess the Fibonacci property (27), with constant $\lambda$ and $\rho$.

Therefore, we have to find a modification of the Fibbonacci property which would be adequate for the $\mu$-oscillator and also for some models that extend it.

\section{Possible ways of generalizing the FP}

Among possible modifications, we could try an extended version of the usual Fibonacci relation (1) obtained merely by adding extra terms. Say we could consider the $k$-term extended (so-called $k$-bonacci) relation of the form (see e.g. $[4,5]$ ):

$$
E_{n}=\alpha_{1} E_{n-1}+\alpha_{2} E_{n-2}+\alpha_{3} E_{n-3}+\ldots+\alpha_{k} E_{n-k} .
$$

For the $\mu$-oscillator, let us check the first extended case of $k=3$ (so-called "Tribonacci relation"). It is proved that the $\mu$-oscillator does not satisfy the Tribonacci relation. The proof goes in complete analogy with the above case of $k=2$, see Proposition 2: by deriving the corresponding system of (now six) equations and then trying to solve that system. As result, we are led to the inconsistency of the system and thus to the conclusion that the $\mu$-oscillator does not satisfy the Tribonacci relation. Moreover, this negative result for the $\mu$-oscillator extends to all other cases $k \geq 3$ of $k$-bonacci relation. 
Next, the most radical possibility would be to search for some nonlinear generalization of (1), say in the form $E_{n+1}=F\left(E_{n}, E_{n-1}\right)$ with $F(x, y)$ an appropriate function [3]. We prefer however to preserve both the linearity and the three-term form of relation. But some price should be payed for such a choice, and the price is nothing but the loss of constant nature of $\lambda$ and $\rho$. In other words, these coefficients inevitably should be $n$-dependent.

\subsection{Quasi-Fibonacci property of $\mu$-deformed oscillator}

We will call "quasi-Fibonacci" oscillators those deformed oscillators the energy spectrum of which satisfy the extended or quasi-Fibonacci (QF) relation involving $\lambda=\lambda(n) \equiv \lambda_{n}$ and $\rho=\rho(n) \equiv \rho_{n}$ :

$$
E_{n+1}=\lambda_{n} E_{n}+\rho_{n} E_{n-1} .
$$

We will show that the $\mu$-deformed oscillator belongs to the set of QF oscillator models. That is, by passing from the Fibonacci property to the QF one, we will prove that it is the QF property which is adequate to the energy spectrum of $\mu$-oscillator.

There exist three different ways of finding $\lambda_{n}$ and $\rho_{n}$ needed to prove the QF property.

\subsubsection{First way of finding $\lambda_{n}, \rho_{n}$ (by the "splitting" ansatz)}

To start with, let us recall that the energy spectrum $E_{n}$ of the deformed oscillator consists of two terms given by the structure function $\varphi(n)$ :

$$
E_{n}=\frac{1}{2}(\varphi(n)+\varphi(n+1)) .
$$

From (29) and (30), using the "splitting", we have the following system of equations:

$$
\left\{\begin{array}{l}
\varphi(n+1)=\lambda_{n} \varphi(n)+\rho_{n} \varphi(n-1), \\
\varphi(n+2)=\lambda_{n} \varphi(n+1)+\rho_{n} \varphi(n) .
\end{array}\right.
$$

Solving the latter system, for $\lambda_{n}$ and $\rho_{n}$ we have

$$
\lambda_{n}=\frac{\varphi(n+1)-\rho_{n} \varphi(n-1)}{\varphi(n)}, \quad \rho_{n}=\frac{\varphi(n+2) \varphi(n)-\varphi^{2}(n+1)}{\varphi^{2}(n)-\varphi(n+1) \varphi(n-1)} .
$$

From (32), using the expression (25) for $\varphi(n)$ of the $\mu$-oscillator, we finally obtain

$$
\begin{aligned}
& \lambda_{n}=2 \frac{(1+\mu(1+2 n))}{(1+2 \mu n)} \cdot \frac{(1+\mu n)}{1+\mu(n+2)}, \\
& \rho_{n}=-\frac{(1+2 \mu(n+1))}{(1+2 \mu n)} \cdot \frac{(1+\mu n)(1+\mu(n-1))}{(1+\mu(n+2))(1+\mu(n+1))} .
\end{aligned}
$$

It is clear that, for consistency, from (33) and (34) in the limit $\mu \rightarrow 0$ we should recover the constants:

$$
\lim _{\mu \rightarrow 0} \lambda_{n}=2, \quad \lim _{\mu \rightarrow 0} \rho_{n}=-1,
$$

i.e., the values $\lambda, \rho$ of the usual harmonic oscillator. Obviously, that is true. Hence we conclude that the $\mu$-oscillator satisfies (29) with (26) and (33), (34), and thus is quasi-Fibonacci one. 


\subsubsection{Second way to find $\lambda_{n}$ and $\rho_{n}$ (by the substitution ansatz)}

Recall that most general form of defining commutation relation looks as [13]

$$
\varphi(N+1)-F(N) \varphi(N)=G(N)
$$

By treating this as the recursion relation and fixing the initial conditions

$$
\varphi(0)=0, \quad \varphi(1)=G(0)=\varepsilon, \quad \varepsilon \in \Re,
$$

the structure function $\varphi(n)$ can be obtained as

$$
\varphi(n)=F(n-1) !\left(\varepsilon+\sum_{j=1}^{n-1} \frac{G(j)}{F(j) !}\right)
$$

where $F(j) !=F(j) F(j-1) \ldots F(1)$ and $F(0) !=1$ (at $\varepsilon=0$ the corresponding formula has been given in [13]).

For our aim, we proceed in analogy with (36)-(38). So we use the ansatz

$$
\rho_{n}=\lambda_{n-1} \quad \text { or } \quad \rho_{n+1}=\lambda_{n} .
$$

With account of this, the QF relation (29) rewrites in the form

$$
E_{n+2}=\lambda_{n+1} E_{n+1}+\lambda_{n} E_{n}
$$

or, for more explicit analogy with (36), in the form

$$
\lambda_{n+1}-\left(-\frac{E_{n}}{E_{n+1}}\right) \lambda_{n}=\frac{E_{n+2}}{E_{n+1}}, \quad n \geq 1 .
$$

Fixing the initial conditions as

$$
\lambda_{0}=\lambda(0)=0, \quad \lambda_{1}=\lambda(1)=\frac{E_{2}}{E_{1}}
$$

we derive for $\lambda_{n}$ the expression

$$
\lambda_{n}=\left(-\frac{E_{n-1}}{E_{n}}\right) ! \sum_{j=0}^{n-1} \frac{E_{j+2} / E_{j+1}}{\left(-E_{j} / E_{j+1}\right) !} .
$$

The coefficient $\rho_{n}$ then follows from (39). In another way, we may use the relation (41) to deduce $\lambda_{n}$ recursively. Recall that the lowest values of $\lambda_{n}$ besides (42), are:

$$
\begin{array}{ll}
\lambda_{2}=-1+\frac{E_{3}}{E_{2}}, & \lambda_{3}=-1+\frac{E_{4}+E_{2}}{E_{3}}, \\
\lambda_{4}=-1+\frac{E_{5}+E_{3}-E_{2}}{E_{4}}, & \lambda_{5}=-1+\frac{E_{6}+E_{4}-E_{3}+E_{2}}{E_{5}},
\end{array}
$$

and the formula for generic $\lambda_{n}$ (which is equivalent to (43), is

$$
\lambda_{n}=-1+\frac{E_{n+1}+E_{n-1}+\sum_{j=2}^{n-2}(-1)^{n-k+1} E_{j}}{E_{n}}=\frac{1}{E_{n}} \sum_{j=2}^{n+1}(-1)^{n-j+1} E_{j} .
$$


Let us remark that instead of the initial condition $\lambda(0)=0$ in eq. (42), we can set more general one, namely

$$
\lambda_{0}=\lambda(0)=c, \quad c=E_{1} / E_{0} .
$$

Then it follows from (40) that

$$
\lambda_{1}=\frac{E_{2}-c E_{0}}{E_{1}}, \quad \lambda_{2}=\frac{E_{3}-E_{2}+c E_{0}}{E_{2}}, \quad \lambda_{3}=\frac{E_{4}-E_{3}+E_{2}-c E_{0}}{E_{3}},
$$

and by applying induction, we arrive at the desired result

$$
\lambda_{n}=\frac{\sum_{k=2}^{n+1}(-1)^{n-k+1} E_{k}+(-1)^{n} c E_{0}}{E_{n}} .
$$

Of course, putting $c=0$ reproduces (44). Using $c=E_{1} / E_{0}$ in (45) the formula (46) takes the form

$$
\lambda_{n}=(-1)^{n+1} \frac{1}{E_{n}} \sum_{k=1}^{n+1}(-1)^{k} E_{k}
$$

which after substitution of (26) yields the desired result:

$$
\begin{aligned}
& \lambda_{n}=\left\{\frac{(-1)^{n}}{1+\mu}+\frac{n+2}{1+\mu(n+2)}\right\} \frac{(1+\mu n)(1+\mu(n+1))}{n(1+\mu(n+1))+(n+1)(1+\mu n)}, \\
& \rho_{n}=\left\{\frac{(-1)^{n-1}}{1+\mu}+\frac{n+1}{1+\mu(n+1)}\right\} \frac{(1+\mu(n-1))(1+\mu n)}{(n-1)(1+\mu n)+n(1+\mu(n-1))} .
\end{aligned}
$$

The solution (48)-(49) of eq. (29) obviously differs from the solution (33)-(34) as the ansatz applied in subsection 3.3.2 is essentially different from the one used in 3.3.1.

\subsubsection{Third (most general) approach for deriving $\lambda_{n}$ and $\rho_{n}$}

We first remark that the formulae derived in the preceding two subsections do not constitute general result. The goal of this subsection is just to find $\lambda_{n}$ and $\rho_{n}$ from the QF relation (29) within most general approach. If we take instead of (29) the system

$$
\left\{\begin{array}{l}
E_{n+1}=\lambda_{n} E_{n}+\rho_{n} E_{n-1}, \\
E_{n+2}=\lambda_{n+1} E_{n+1}+\rho_{n+1} E_{n}
\end{array}\right.
$$

(as 2 equations with 4 unknowns) we cannot calculate $\lambda$ and $\rho$ as before, see (17)-(18).

To find explicitly $\lambda_{n}$ and $\rho_{n}$ satisfying (29) along with (26), that is, the relation

$$
\frac{n+2}{1+\mu(n+2)}=\left(\lambda_{n}-1\right) \frac{n+1}{1+\mu(n+1)}+\left(\lambda_{n}+\rho_{n}\right) \frac{n}{1+\mu n}+\rho_{n} \frac{n-1}{1+\mu(n-1)},
$$

we now follow more general scheme: we replace (51) with the pair of equations

$$
\frac{2 n+2}{1+\mu(n+2)}=\left(\lambda_{n}-1\right) \frac{n+1}{1+\mu(n+1)}+\frac{K(n ; \mu)(n+1)}{1+\mu(n+1)},
$$




$$
\frac{-n}{1+\mu(n+2)}=\left(\lambda_{n}+\rho_{n}\right) \frac{n}{1+\mu n}+\rho_{n} \frac{n-1}{1+\mu(n-1)}-\frac{K(n ; \mu)(n+1)}{1+\mu(n+1)} .
$$

Note that, the unspecified function $K=K(n ; \mu)$ introduced here, due to its arbitrariness, encapsulates the non-uniqueness of splitting (51) into two equations and thus provides equivalence of (51) with the pair (52)-(53). Indeed, adding (52) and (53) yields (51). Or, isolating the last term (with $K(n ; \mu))$ in (52) and the identical term in (53), we get the equivalence: $(51) \leftrightarrow(52) \&(53)$.

Now, (52)-(53) are easily solved for $\lambda_{n}$ and $\rho_{n}$ that yields the desired general result

$$
\begin{aligned}
& \lambda_{n}=1-K(n ; \mu)+2 \frac{1+\mu(n+1)}{1+\mu(n+2)}, \\
& \rho_{n}=\frac{1+\mu(n-1)}{1+2(n-1)(1+\mu n)}\left\{K(n ; \mu) \frac{2(n+1)(1+\mu n)-1}{1+\mu(n+1)}-\frac{4 n(1+\mu(n+1))}{1+\mu(n+2)}\right\} .
\end{aligned}
$$

Remark 1. Various choices of the function $K(n ; \mu)$ are of interest. Say, by properly specified choice $K=K^{(1)}$ (respectively $K=K^{(2)}$ ), we can reproduce from (54)-(55) the expressions (33), (34) in subsection 3.3.1 (respectively the formulae (48), (49) in subsection 3.3.2). It is easy to verify that the corresponding choices of $K(n ; \mu)$ are:

$$
\begin{aligned}
K^{(1)} & =\frac{1+3 \mu n(1+2 \mu)+2 \mu\left(\mu n^{2}+1\right)}{(1+2 \mu n)(1+\mu(n+2))} \\
K^{(2)} & =\frac{\mu}{1+\mu(n+2)}+\frac{(-1)^{n+1}(1+\mu n)(1+\mu(n+1))}{(1+\mu)\{n(1+\mu(n+1))+(n+1)(1+\mu n)\}}+ \\
& +\frac{(n+1)\{3 \mu n+(5 n+1)(1+\mu n)\}}{(1+\mu(n+2))\{n(1+\mu(n+1))+(n+1)(1+\mu n)\}} .
\end{aligned}
$$

From viewing the quasi-Fibonacci relation as "locally-Fibonacci" property, see end of Introduction, it follows that the (auxiliary) function $K(n ; \mu)$, introduced in order to account the non-uniqueness of replacing (51) by the pair (52)\&(53) plays the role somewhat resembling gauge freedom in gauge theories: the quantities $\lambda_{n}$ and $\rho_{n}$ depend on $K(n ; \mu)$ while the energies $E_{n}$ do not, see (26) and (50)-(51).

Remark 2. As mentioned above, the usual harmonic oscillator is Fibonacci oscillator for which $\lambda=2, \rho=-1$. Its structure function and energy spectrum obviously stem from (25), (26) if $\mu \rightarrow 0$. However, sending $\mu \rightarrow 0$ in the formulas (54)-(55) gives

$$
\lambda_{n}=3-K(n ; 0), \quad \rho_{n}=\frac{K(n ; 0)(2 n+1)-4 n}{2 n-1},
$$

i.e., there is a residual dependence on $n$ if $K(n ; 0) \neq 1$. This looks as a kind of surprise: due to $n$-dependent $\lambda_{n}, \rho_{n}$ the usual oscillator can also be treated as quasi-Fibonacci oscillator (satisfying (29)). Only if $K(n ; 0)=1$ is put, we get $\lambda=2, \rho=-1$, and the usual oscillator becomes genuine Fibonacci oscillator.

Concerning the last phrase it is worth to add the following. ¿From (56) and (57) at $\mu \rightarrow 0$ we have: $\left.K^{(1)}\right|_{\mu=0}=1$, but $\left.K^{(2)}\right|_{\mu=0} \neq 1$. This explains the fact that $\lambda_{n}$ and $\rho_{n}$ from (33)-(34) do lead at $\mu \rightarrow 0$ to the values $\lambda=2$ and $\rho=-1$ of the ordinary oscillator, while those in (48)-(49) do not (the reason is the very ansatz $\rho_{n}=\lambda_{n-1}$ in (39)). 
Remark 3. The extension based on replacing the constant $\lambda, \rho$ with $\lambda_{n}=\lambda(n)$ and $\rho_{n}=\rho(n)$ is of principal value for the QF property. However, for the true QF property to be valid it is in fact enough that only one of the coefficients depends on $n$, the other being constant. For instance, consider the case: $\lambda_{n}=\lambda(n)$ and $\rho=-1$ for the considered $\mu$-oscillator. Imposing $\rho=-1$ in (55) yields the specified $K(n ; \mu)$-function:

$$
K(n ; \mu)_{\rho=-1}=\frac{(1+\mu(n+1))\left\{2 \mu^{2} n^{2}(3 n+1)+4 \mu n(3 n+2 \mu)+\mu(n-2)+6 n-1\right\}}{(1+\mu(n-1))(1+\mu(n+2))\{2(n+1)(1+\mu n)-1\}}
$$

With this particular $K$-function, we find the $\lambda_{n}$ related to $\rho=-1$ :

$$
\lambda(n)_{\rho=-1}=1-\frac{(1+\mu(n+1))\left\{10 \mu n^{2}(2+\mu n)+(1-4 \mu)(3 \mu n+1)+12 n\right\}}{(1+\mu(n-1))(1+\mu(n+2))\{2(n+1)(1+\mu n)-1\}} .
$$

It is worth to mention that similar form of the QF relation, with $\rho=-1$ and certain $\lambda=\lambda(n)$, appears in [30], where the members of the QF sequence therein have the physical sense of (the Fourier transform of) the oscillation amplitude of dipole with number $n$ in the non-periodic chain of dipoles.

Likewise, we may impose $\lambda=2$ and then find from (54)-(55) the relevant $K$-function and the corresponding $\rho=\rho_{n}$ :

$$
\begin{aligned}
& K(n ; \mu)_{\lambda=2}=2 \frac{(1+\mu(n+1))}{(1+\mu(n+2))}-1, \\
& \rho(n)_{\lambda=2}=-\frac{1+\mu(n-1)}{1+2(n+1)(1+\mu n)} \cdot \frac{n(2+5 \mu)+4 \mu n(n+\mu)+2 \mu^{2} n^{2}(n+3)-1}{(1+\mu(n+1))(1+\mu(n+2))} .
\end{aligned}
$$

Remark 4. Let $K$ in (54), (55) be equal to zero. Then $\lambda_{n}=\frac{P_{1}}{Q_{1}}$, where $P_{1}$ and $Q_{1}$ are linear in $n$, while $\rho_{n}=\frac{P_{3}}{Q_{3}}$ where $P_{3}$ and $Q_{3}$ are cubic in $n$. At $K=1$, however, $\lambda_{n}$ is again "linear/linear" expression in $n$, but $\rho_{n}$ is the "quadratic/quadratic" one. Let us compare the structure of the coefficients $\lambda_{n}$ and $\rho_{n}$ which were obtained in different ways, see subsections 3.3.1-3.3.3. While $\lambda_{n}, \rho_{n}$ in (33), (34) are of the form: $\lambda_{n}=\frac{P_{2}^{(1)}}{Q_{2}^{(1)}}$ and $\rho_{n}=\frac{\widetilde{P}_{3}^{(1)}}{\widetilde{Q}_{3}^{(1)}}$, the structure seen in (48), (49) looks as $\lambda_{n}=\frac{P_{3}^{(2)}}{Q_{3}^{(2)}}$ and $\rho_{n}=\frac{\widetilde{P}_{3}^{(2)}}{\widetilde{Q}_{3}^{(2)}}$. Likewise, an appropriate choice of the function $K(n ; \mu)$ in the general expressions (54), (55), yields another particular case for $\lambda_{n}, \rho_{n}$ such that they also both take the form $\frac{P_{3}^{(3)}}{Q_{3}^{(3)}}$. Indeed, the choice

$$
K=\frac{(1+\mu(n+1))(1+2(n-1)(1+\mu n))}{(1+\mu(n+2))(-1+2(n+1)(1+\mu n))}
$$

again leads to the "cubic/cubic" expressions for both $\lambda_{n}$ and $\rho_{n}$ :

$$
\begin{aligned}
& \lambda_{n}=1+\frac{1+\mu(n+1)}{1+\mu(n+2)} \cdot \frac{3+2(1+\mu n)(3 n+1)}{1-2(n+1)(1+\mu n)}, \\
& \rho_{n}=-\frac{1+\mu(n-1)}{1+2(n-1)(1+\mu n)} \cdot \frac{1+2 n(1+\mu(n+3))}{1+\mu(n+2)} .
\end{aligned}
$$

To end this section, we find it instructive to tabulate $\lambda_{n}$ and $\rho_{n}$ from (54)-(55) for low values of $n$, together with $E_{n}$, at fixed $K(n ; \mu)=1$, see Table 1 . 
Table 1. Explicit form of $\lambda_{n}$ and $\rho_{n}$ at low values of $n$.

\begin{tabular}{c|c|l|l}
\hline \hline$n$ & $\lambda_{n}$ & $\rho_{n}$ & $E_{n}$ \\
\hline \hline 0 & $\lambda_{0}=\frac{2+2 \mu}{1+2 \mu}$ & $\rho_{0}=\frac{\mu-1}{1+\mu}$ & $E_{0}=\frac{1}{2(1+\mu)}$ \\
\hline 1 & $\lambda_{1}=\frac{2+4 \mu}{1+3 \mu}$ & $\rho_{1}=\frac{3+4 \mu}{1+2 \mu}-\frac{4(1+2 \mu)}{1+3 \mu}$ & $E_{1}=\frac{1}{2(1+\mu)}+\frac{1}{1+2 \mu}$ \\
\hline 2 & $\lambda_{2}=\frac{2+6 \mu}{1+4 \mu}$ & $\rho_{2}=\frac{1+\mu}{3+4 \mu}\left(\frac{5+12 \mu}{1+3 \mu}-\frac{8(1+3 \mu)}{1+4 \mu}\right)$ & $E_{2}=\frac{1}{1+2 \mu}+\frac{3}{2(1+3 \mu)}$ \\
\hline 3 & $\lambda_{3}=\frac{2+8 \mu}{1+5 \mu}$ & $\rho_{3}=\frac{1+2 \mu}{5+12 \mu}\left(\frac{7+24 \mu}{1+4 \mu}-\frac{12(1+4 \mu)}{1+5 \mu}\right)$ & $E_{3}=\frac{3}{2(1+3 \mu)}+\frac{2}{1+4 \mu}$ \\
\hline 4 & $\lambda_{4}=\frac{2+10 \mu}{1+6 \mu}$ & $\rho_{4}=\frac{1+3 \mu}{7+24 \mu}\left(\frac{9+40 \mu}{1+5 \mu}-\frac{16(1+5 \mu)}{1+6 \mu}\right)$ & $E_{4}=\frac{2}{1+4 \mu}+\frac{5}{2(1+5 \mu)}$ \\
\hline 5 & $\lambda_{5}=\frac{2+12 \mu}{1+7 \mu}$ & $\rho_{5}=\frac{1+4 \mu}{9+40 \mu}\left(\frac{11+60 \mu}{1+6 \mu}-\frac{20(1+6 \mu)}{1+7 \mu}\right)$ & $E_{5}=\frac{5}{2(1+5 \mu)}+\frac{3}{1+6 \mu}$ \\
\hline
\end{tabular}

\section{Mixed cases of deformed oscillators}

It is possible to construct new models of deformed oscillators which are quasiFibonacci extensions of a particular Fibonacci oscillator of Section 2, by combining the latter with the $\mu$-oscillator which plays the role of the basic quasi-Fibonacci "building block". Clearly, the procedure brings in some additional deformation parameters. On this way we naturally obtain new models with two deformation parameters $\mu$ and $q$ : say, the mixed $\mu-A C$ case, and also the mixed $\mu-B M$ and $\mu-T D$ cases. However, since the models of $q$-deformed oscillators are contained as particular cases in the $p, q$-family, see sections 2.1-2.3, it is useful to start with the three-parameter, mixed $(\mu ; p, q)$-family of models.

\section{The three-parameter "mixed" family of $(\mu ; p, q)$-deformed oscillators}

This family of deformed oscillator models arises due to combining the $p, q$-oscillator with the $\mu$-oscillator and is given by the structure function

$$
\varphi_{\mu, p, q}(n)=\frac{[n]_{p, q}}{1+\mu n}, \quad[n]_{p, q} \equiv \frac{p^{n}-q^{n}}{p-q} .
$$

Of course, the choice (61) of "mixed" deformation is not unique: for the "mixed" structure function one could also use, say, $\psi_{\mu, p, q}(n)=\frac{[n]_{p, q}}{1+\mu[n]_{p, q}}$ or $\chi_{\mu, p, q}(n)=\left[\frac{n}{1+\mu n}\right]_{p, q}$. Our choice however (i) is simpler from the viewpoint of further use in applications like that in [31] and (ii) better correlates with the ideas of [10].

So we expect that this $(\mu ; p, q)$-oscillator, with the energy spectrum

$$
E_{n}=\frac{1}{2}\left(\varphi_{\mu, p, q}(n+1)+\varphi_{\mu, p, q}(n)\right)
$$

obeys the QF relation. 
It is clear that the $(\mu ; p, q)$-deformed family cannot satisfy pure Fibonacci relation, because of its $\mu$-component as carrier of the QF property; only if $\mu=0$ it reduces to the pure $(p, q)$-oscillator for which the status of Fibonacci oscillator is recovered.

So, consider the $(\mu ; p, q)$-oscillator as quasi-Fibonacci one. The QF property of it will be certified if the explicit expressions for $\lambda_{n}$ and $\rho_{n}$ are found such that the QF relation (29) holds true:

$$
\begin{aligned}
& \frac{[2]_{p, q}[n+1]_{p, q}-p q[n]_{p, q}}{1+\mu(n+2)}+\frac{[n+1]_{p, q}}{1+\mu(n+1)}= \\
& =\lambda_{n}\left(\frac{[n+1]_{p, q}}{1+\mu(n+1)}+\frac{[n]_{p, q}}{1+\mu n}\right)+\rho_{n}\left(\frac{[n]_{p, q}}{1+\mu n}+\frac{[n-1]_{p, q}}{1+\mu(n-1)}\right) .
\end{aligned}
$$

Note that in the LHS the identity:

$$
[n+2]_{p, q}=[2]_{p, q}[n+1]_{p, q}-p q[n]_{p, q}
$$

has been used. Instead of (62) we may equivalently exploit the following two relations:

$$
\begin{aligned}
& \left(\lambda_{n}-1\right) \frac{[n+1]_{p, q}}{1+\mu(n+1)}=\frac{[2]_{p, q}[n+1]_{p, q}}{1+\mu(n+2)}-K \frac{[n+1]_{p, q}}{1+\mu(n+1)}, \\
& \left(\lambda_{n}+\rho_{n}\right) \frac{[n]_{p, q}}{1+\mu n}+\rho_{n} \frac{[n-1]_{p, q}}{1+\mu(n-1)}=\frac{-p q[n]_{p, q}}{1+\mu(n+2)}+K \frac{[n+1]_{p, q}}{1+\mu(n+1)} .
\end{aligned}
$$

Let us emphasize that the arbitrary function $K=K(n ; \mu, p, q)$ involves both the variable $n$ and the three deformation parameters. Like before, it is the unspecified function $K(n ; \mu, p, q)$ which guarantees the equivalence with (62). From the pair of equations, using $[2]_{p, q}=p+q$ as implied by (61), the desired solution does follow, namely

$$
\begin{aligned}
& \lambda_{n} \equiv \lambda_{n}(\mu, p, q)=1-K+[2]_{p, q} \frac{1+\mu(n+1)}{1+\mu(n+2)} \\
& \rho_{n} \equiv \rho_{n}(\mu, p, q)=\frac{1+\mu(n-1)}{[n]_{p, q}(1+\mu(n-1))+[n-1]_{p, q}(1+\mu n)} \times \\
& \times\left\{K\left([n]_{p, q}+\frac{[n+1]_{p, q}(1+\mu n)}{1+\mu(n+1)}\right)-[n]_{p, q}\left(1+\frac{[2]_{p, q}(1+\mu(n+1))+q p(1+\mu n)}{1+\mu(n+2)}\right)\right\}
\end{aligned}
$$

This constitutes our general result for the $(\mu ; p, q)$-deformed oscillators. From this, by fixing $K(n ; \mu, p, q)$ we can generate for $\lambda_{n}$ and $\rho_{n}$ various special expressions.

Let us consider some special cases of (64), (65), with fewer deformation parameters.

1) If $p=q=1$ and $\mu \neq 0$, it is easily checked that one recovers the expressions (54), (55) in Sec. 3.3 for $\lambda(n)$ and $\rho(n)$ of the pure $\mu$-oscillator, the latter being the carrier of QF property.

2) If we put $\mu=0$ in (64), (65), we have:

$$
\lim _{\mu \rightarrow 0} \lambda_{n}(\mu, p, q)=\lambda(p, q)=\lambda=1-K(n ; p, q)+[2]_{p, q},
$$




$$
\lim _{\mu \rightarrow 0} \rho_{n}(\mu, p, q)=\rho(p, q)=\rho=\frac{K(n ; p, q)([n+1]+[n])-[n]\left(1+p q+[2]_{p, q}\right)}{[n]_{p, q}+[n-1]_{p, q}} .
$$

In the latter expressions $\lambda$ and $\rho$ still depend on $n$. However, at $K=1$ we recover $\lambda=[2]_{p, q}$ and $\rho=-p q$ of the pure $(p, q)$-oscillator in Sec.2. The cancelation of the $n$-dependence in $\lambda$, see $K(n ; p, q)$, and in $\rho$, follows by applying the identity (63).

3) Let $p=q^{-1}$ and $q \rightarrow 1$. The corresponding values of the coefficients then read

$$
\begin{aligned}
& \lambda_{n}=1-K(n ; 1,1)+\frac{2(1+\mu(n+1))}{1+\mu(n+2)}, \\
& \rho_{n}=\frac{1+\mu(n-1)}{1+2(n-1)(1+\mu n)}\left(K(n ; 1,1) \frac{2(n+1)(1+\mu n)-1}{1+\mu(n+1)}-\frac{4 n(1+\mu(n+1))}{1+\mu(n+2)}\right)
\end{aligned}
$$

and if moreover $K=1$, we have from the letter:

$$
\begin{aligned}
& \left.\lambda_{n}\right|_{K=1}=2 \frac{1+\mu(n+1)}{1+\mu(n+2)}, \\
& \left.\rho_{n}\right|_{K=1}=\frac{1+\mu(n-1)}{1+2(n-1)(1+\mu n)}\left(1-2 n+\mu\left(\frac{4 n}{1+\mu(n+2)}-\frac{n+1}{1+\mu(n+1)}\right)\right) .
\end{aligned}
$$

4) Putting $\mu=0, p=q=1$ in (64)-(65), for $\lambda_{n}$ and $\rho_{n}$ yields

$$
\left.\lambda_{n}\right|_{\mu=0}=3-K,\left.\quad \rho_{n}\right|_{\mu=0}=\frac{K(2 n+1)-4 n}{2 n-1} .
$$

see Remark 2 for more details.

As mentioned at the beginning of this section, since we have at our disposal the explicit formulae (64), (65) for the 3-parameter $(\mu ; p, q)$-family, we immediately get the corresponding results for the three distinguished two-parameter cases. Let us illustrate this (recall (8), (10), and $[n]_{T D} \equiv n q^{n-1}$ for the AC, BM and TD cases correspondingly):

- let $p \rightarrow 1$. In this case, $\varphi_{\mu, A C}(n)=\frac{[n]_{A C}}{1+\mu n} \quad$ (mixed $\mu$-AC case);

- put $p=q^{-1}$. In this case $\varphi_{\mu, B M}(n)=\frac{[n]_{B M}}{1+\mu n} \quad$ (mixed $\mu$-BM case);

- put $p=q . \quad$ In this case $\varphi_{\mu, T D}(n)=\frac{[n]_{T D}}{1+\mu n} \quad$ (mixed $\mu$-TD case).

We end with the comment on the hybrid case of six parameters: the $(\mu ; p, q, \alpha, \beta, l)$-case.

Remark 5. All the above treatment carried out for the three-parameter $(\mu ; p, q)$ family of deformed oscillators can be easily extended to the case of six-parameter family which combines the 5-parametric case (16) and the formula (26) of the $\mu$-oscillator case. Also, one can put $l=\alpha$ in order to have unambiguous definition of the structure function, consistent with the relation $E_{n}=\frac{1}{2}(\varphi(n)+\varphi(n+1))$.

\section{Conclusions and outlook}

In this paper we dealt with two essentially different classes of deformed oscillators: the Fibonacci class and the (much more rich) quasi-Fibonacci class. As our first result, we have proven that besides the well-studied 2-parameter family of $p, q$-deformed oscillators known [2] as Fibonacci oscillators, there exists more general, with three 
additional parameters, the $(p, q, \alpha, \beta, l)$-family of deformed oscillators which does also belong to the Fibonacci class. What is rather unexpected, the coefficients $\lambda$ and $\rho$ for the 5-parametric family of deformed oscillators depend on $p, q$ (as in the $p, q$-deformed case) and else only on one parameter $\alpha$, from the remaining three parameters.

On the other hand, according to our second main result reflected in Proposition 2, the $\mu$-oscillator [10] is not in Fibonacci class, but belongs to the class of QF oscillators whose basic feature is that the sequence of values of energy levels obeys the QF relation, being as well linear and three-term (or two-step) one, but involving the non-constant coefficients $\lambda_{n}$ and $\rho_{n}$ which depend besides the deformation parameter $\mu$ also on the number $n$ of the energy level $E_{n}$.

The peculiar feature of QF oscillators is that the $n$-dependent coefficients $\lambda_{n}$ and $\rho_{n}$ are obtained non-uniquely, as it was demonstrated with the three different methods of solving the eq.(29). While the first two ways lead to partial solutions, the third method is general one due to the (arbitrary) function $K(n ; \mu)$ being involved.

We have shown that the $\mu$-oscillator is not the unique one possessing the QF property. Indeed, it can be utilized as a basic ingredient for constructing other families of QF deformed oscillators which have additional deformation parameter(s). An example of the three-parameter family of $(\mu ; p, q)$-deformed oscillators obtainable through combining the $p, q$-oscillator with the $\mu$-oscillator has been considered. From that, a particular two-parameter families (the $\mu, q$-oscillator as the Arik-Coon \& the $\mu$-type hybrid, the BM \& the $\mu$-type hybrid, and the TD \& the $\mu$-type hybrid) of deformed oscillators naturally follow. We believe our results provide the basis for constructing in a regular manner numerous new models of multi-parameter deformed nonlinear oscillators belonging to different extensions of the Fibonacci class, in particular, those possessing the quasi-Fibonacci property. It is also worth to note that interesting classes of quasi-Fibonacci oscillators have been explored in our recent paper [5]. The polynomially deformed non-Fibonacci oscillators treated therein are rather amusing as they can be viewed in three different ways: 1) as quasi-Fibonacci oscillators; 2) as the oscillators obeying inhomogeneous Fibonacci relation; 3) as $k$-bonacci oscillators. Unlike the classes just mentioned, the $\mu$-oscillator studied in the present paper along with its direct extensions admit only the quasi-Fibonacci type (way) of description.

For each Fibonacci oscillator there exist diverse quasi-Fibonacci extensions, see e.g. the note below (61) and Remark 5. This fact, all that is said in the preceding paragraph, and the results of Sec.3, Sec.4 clearly demonstrate that the class of QF models based on the novel concept of quasi-Fibonacci oscillators is really rich and worth of detailed study. Although exploring of physical applications of quasi-Fibonacci oscillators is at the very beginning, we may quote the recent work [31] on the $\mu$-Bose gas model employing $\mu$ oscillators. Let us finally note that in view of existing deformed fermionic Fibonacci oscillators [32] it is worth to study, along the lines of the present paper, possible models of fermionic quasi-Fibonacci oscillators, as yet another class.

\section{Acknowledgement}

The authors are thankful to I.M. Burban for interesting discussions, and to the referees for valuable remarks and suggestions which led to improved presentation. This research was partially supported by the Grant 29.1/028 of the State Foundation of Fundamen- 
tal Research of Ukraine and by the Special Program of the Division of Physics and Astronomy of the NAS of Ukraine.

\section{References}

[1] Chakrabarti R and Jagannathan R 1991 J. Phys. A: Math. Gen. 24 L711

[2] Arik M et al 1992 Z. Phys. C 55 89-95

[3] de Souza J, Curado E M F and Rego-Monteiro M A 2006 J. Phys. A: Math. Gen. 39 10415-10425

[4] Schork M 2007 J. Phys. A: Math. Theor. 40, 4207-4214

[5] Gavrilik A M and Rebesh A P 2010 J. Phys. A: Math. Theor. 43095203 (15pp)

[6] Chung W-S, Chung K-S and Nam S-T 1993 Phys. Let. A 183 363-370

[7] Borzov V V, Damaskinsky E V and Yegorov S B 1997 Zap. Nauch. Semin. LOMI 24580

[8] Mizrahi S S, Camargo Lima J P and Dodonov V V 2004 J. Phys. A: Math. Gen. 373707

[9] Burban I M 2007 Phys. Let. A 366 308-314

[10] Jannussis A 1993 J. Phys. A: Math. Gen. 26 L233-L237

[11] Wei Shao-Wen, Ran Li, Yu-Xiao and Ji-Rong Ren Quantization of black hole entropy from quasinormal modes, arxiv:0901.0587 [hep-th].

[12] Man'ko V I, et al. 1997 Phys. Scripta 55528

[13] Meljanac S, Mileković M and Pallua S 1994 Phys. Lett. B 328 55-59

[14] Bonatsos D and Daskaloyannis C 1999 Prog. Part. Nucl. Phys. 43537

[15] Arik M and Coon D D 1976 J. Math. Phys. 17524

[16] Biedenharn L C 1989 J. Phys. A: Math. Gen. 22 L873

[17] Macfarlane A J 1989 J. Phys. A: Math. Gen. 224581

[18] Adamska L V and Gavrilik A M 2004 J. Phys. A: Math. Gen. 374787

[19] Gavrilik A M 2006 SIGMA 212 (paper 074) arxiv: hep-ph/0512357

[20] Crnugelj J, Martinis M and Mikuta-Martinis V 1994 Phys. Rev. A 50 1785-1791

[21] Algin A 2008 J. Stat. Mech: Theor. Exp. 10009

[22] Algin A 2009 J. Stat. Mech: Theor. Exp. 04007 
[23] Ben Geloun J, Govaerts J and Hounkonnou M N 2007 Eur. Phys. Lett. 8030001 (6pp)

[24] Gavrilik A M and Rebesh A P 2008 Ukr. J. Phys. 53586

[25] Gavrilik A M and Rebesh A P 2008 Mod. Phys. Lett A 23921

[26] Odaka K, Kishi T and Kamefuchi S 1991 J. Phys. A: Math. Gen. 24 L591

[27] Chaturvedi S, Srinivasan V and Jagannathan R 1993 Mod. Phys. Lett. A 83727

[28] Gavrilik A M and Rebesh A P 2007 Mod. Phys. Lett. A 22949

[29] Arik M, Atakishiyev N M and Wolf K B 1999 J. Phys. A 32 L371.

[30] Luca Dal Negro and Ning-Ning Feng 2007 Optics Express 15 No.22, 14396-14403

[31] Gavrilik A M and Rebesh A P, Intercepts of the momentum correlation functions in $\mu$-Bose gas model and their asymptotics (submitted).

[32] Algin A, Arik M and Arikan A S 2002 Eur. Phys. J. C 200225487 